\title{
Errata
}

Due to an error in typesetting, pages 117-125 from Volume 3 Number 2 have been reprinted. We apologize to the author. 
We thus can write:

(define append (lambda ( $x y$ ) ; a function definition

(cond ( (null? $x) y$ )

$(t(\operatorname{cons}(\operatorname{car} x)$ (append $(\operatorname{cdr} x) y))))))$

$\rightarrow$ append

(append '(a) '(b c)) ; a function application

$\rightarrow(\mathrm{a} \quad \mathrm{b}$ c) $)$; returns a term

(define appendr (clause ('() ? $\mathrm{x} ? \mathrm{x})$ )) ; a predicate definition

$\rightarrow$ appendr ; involving two clauses

(define appendr (clause ((cons ?a ?x) ?y (cons ?a ?z))

(appendr ?x ?y ?z)))

$\rightarrow$ appendr

(appendr '(a) '(b c) ?x) ; a predicate application

$\rightarrow((\operatorname{appendr}(\mathrm{a})(\mathrm{b} c)(\mathrm{a} \quad \mathrm{b} c)))$; returns a relation

(appendr ?x ?y '(a b c)) ; a predicate application

$\rightarrow((\operatorname{appendr}()(\mathrm{a} b \mathrm{c})(\mathrm{a} b \mathrm{c}))$; returns a relation (appendr (a) ( $b c)(a b c)$ )

(appendr (a b) (c) (a b c))

(appendr $(a b c)()(a b c)))$

Predicate appendr can also be defined as a one clause predicate using function append as follows:

(define appendr (clause (?x ?y (append ?x ?y))))

With this second definition, the application

(appendr '(a) '(b c) ?x)

will return the same relation as above, provided that the evaluation of arguments proceeds from left to right, interleaved with unification, thus allowing 
the computation of (append ?x ?y) to take place after the unification of ?x with' (a) and of ?y with' ( $b$ c) (i.e. at the unification level rather than at the resolvent level).

On the other hand, in the application

(appendr ?x ?y ' (a b c))

function append would try to access unbound variables. As in any such case in standard Scheme, the evaluator would signal an access to an unbound variable, and give up further evaluation.

\subsection{Comparison With Related Works}

It is worthwhile at this point to compare our proposal with related existing works.

In Robinson and Sibert's original work, predicates are not applicable objects of their own, and their application follow some predefined function applications. Higher order properties of functional languages are not considered. In order to allow term-level integration, their unification algorithm incorporates a reduction process allowing to simplify functional terms containing variables. As pointed out in [4], this will require access to all subterms of terms, and thus can be very costly.

In the work by Srivastava and Alii [8], predicates are pseudo-applicable objects, called logical procedures, wich can be invoked with a call macro. Logical procedures themselves are macros which expand into Scheme functions. Higher order properties of Scheme are preserved, but term-level integration is not considered in their model.

In Hayne's complete functional embedding maintaining higher order properties [5], predicates, called relations, are truly applicable objects, represented as functions. Consequently, as in any function application, predicate arguments get evaluated before application. In other words, the computation of functions takes place at the resolvent rather than at the unification level, which is a limitation to term-level integration.

The comparison with the recent work by Thrift [9] is instructive of the differences which exists between Scheme and Common Lisp. In order to support first class logical variables and relations within Common Lisp, Thrift has to resort to a relation calling mechanism analogous to the one for calling functions. If higher order properties are thus preserved, the direct mixing of terms is not possible, and a call to an interface is required to bind logical variables. On the other hand, standard Prolog compilation techniques are applicable, thus enabling high-speed performance. 
With regard to our own previous model [2], the bindings for both logical and functional variables now form a single environment, expressions get instantiated when evaluated, and arguments are evaluated at the unification rather than at the resolvent level, all things accounting for a tighter integration.

\section{The Computation Model}

Following Haynes's taxonomy [5], our computational model can be described as a functional environment embedding of logic programming into Scheme. Two different computational models thus coexist within a single framework, where they share a common environment.

The main issues that one faces in such models can be characterized as follows:

- how should logical variables be distinguished from ordinary variables?

- how should predicates (i.e the objects clause expressions evaluate to) be distinguished from ordinary function closures?

- how to express the corresponding extended model of evaluation?

\subsection{Logical Variables}

The properties of logical variables can be summarized as follows:

logical variables, whose scope is limited to the clause in which they appear and which are initially unbound, can be referenced (e.g. passed or returned) when unbound, and can become bound by unification.

In [5], this is enforced by listing, for each clause, the identifiers which must be associated with logical variables that are initially unbound, these variables being themselves represented by a reference to a unique value denoting "unbound".

Following [1], we use the prefix ' ?"' for identifiers which must initially evaluate to unbound logical variables. In order to enforce the proper name-binding discipline required by term-level integration, unbound logical variables themselves (which may become bound later on) are treated as first class lexically scoped objects, and thus are represented by a special type of closure, namely logical variable closures. 


\section{Example}

Consider the predicates $\mathbf{p}$ and $\mathbf{q}$ defined as

(define $p($ clause $(? x)(q(c d r ? x))))$

(define q (clause $('(b \quad c)))$ )

and the application

$(\mathrm{p}($ cons 'a ? $\mathrm{x}))$

$\rightarrow((p(a b c)))$

The actual argument (cons 'a ? $\mathrm{x}$ ) will evaluate to the list (a. $[? \mathrm{x}]$ ), where $[? \mathrm{x}]$ represents the closure associating the top level variable ? $\mathrm{x}$ and its environment. After the unification of this actual argument with its corresponding formal parameter, the local variable ? $x$ in the clause for $p$ will be bound to this list. After the cdr computation, the actual argument for $q$ will evaluate to the closure $[? \mathrm{x}]$, where $? \mathrm{x}$ refers to the top level variable. Finally, after the unification of this closure with $(b c)$, the top level variable ?x will be bound to $(\mathrm{b} c)$.

\subsection{Predicates}

Turning now to predicates, they certainly could be represented as function closures (and therefore, predicate applications evaluated as function applications, as they are in [5]) : as pointed out above, their arguments would then be evaluated at the resolvent level, instead of the unification level, which limits term-level integration. Hence a third type of closures, namely clause closures, which are the objects individual clause expressions evaluate to, have been introduced. Multiple clauses predicates, obtained by successive evalutions of clauses (as in the first example for appendr in Section 1.3) will then be given by introducing successive clause closures within a single object.

We must finally deal with the fact that applications of undefined predicates, unlike that of undefined fonctions, are meaningful, returning an empty relation. Any unbound variable should be acceptable to represent such undefined predicates. 


\section{Example}

( $p$ ? $x$ ) ; undefined predicate application if $p$ is unbound

$\rightarrow()$; returns an empty relation

In order to get values for them, we shall allow ordinary unbound variables to evaluate to logical variable closures: if $\mathrm{x}$ is unbound, it will thus evaluate to $[? \mathrm{x}]$, just as ? $\mathrm{x}$ does.

N.B. While this last point may seem questionable, it does bring some natural expressiveness into the model, by allowing ordinary unbound variables to become bound by unification in predicate applications:

\section{(define p (clause ('a)))}

$\rightarrow p$

$(\mathrm{p} x)$

$\rightarrow\left(\left(\begin{array}{ll}p & a\end{array}\right)\right)$; if $x$ is unbound

The possibility of having indistinguishable functional and logical variables is advocated in [8]. We do not go that far, in order to easily distinguish local variables in clauses and global variables of the same name. In our model, $\mathrm{x}$ and $\mathrm{x}$ are still separate variables, even when $\mathrm{x}$ evaluates to $[? \mathrm{x}]$. Similarly, ?y may evaluate to $[? \mathrm{x}]$, if ?y has been unified with $? \mathrm{x}$, and still be a different variable. Properly, we distinguish between "variables", which in Scheme are identifiers which may name locations, and "logical variable closures", which, like other closures, are objects which may be stored in these locations. Among all variables (i.e. all identifiers that are not syntactic keywords), we distinguish between logical variables (i.e identifiers prefixed with ?), which are always local and initially bound to logical variable closures, and ordinary variables, which can be global and are bound to such closures when no other bindings are effective within their scope.

\subsection{The Extended Evaluation Model}

We are thus led to a model of evaluation comprising three types of operator, namely functional operators, containing a function closure, defined logical operators, containing one or more clause closures, and undefined logical operators, represented by a variable closure. Extending Abelson and 
Sussman's environment model of evaluation for Scheme, as embodied in the rules given in Section 1.3, our own model can now be summarized as follows:

- to evaluate an application (i.e any coumpound expression which is not a special form), first evaluate its operator part, giving an operator, and

- if this operator is functional, then evaluate the operand part (i.e. the application arguments), giving the actual arguments, and apply the function closure to the actual arguments

- else, if it is a defined logical operator, then consider in turn each of its clause closures; for all head arguments of a given clause, from left to right, alternate the evaluation of a head argument and the corresponding application argument with the unification of the resulting pair of values: if the two argument lists can be so unified, then apply the corresponding clause closure

- else, if it is an undefined logical operator, then return an empty list

- to apply a function closure to a set of arguments, evaluate the body of the function in a new environment, constructed by extending the environment part of the closure by a frame in which the formal parameters are bound to the actual arguments

- to apply a clause closure whose head arguments have been unified with application arguments, evaluate the body of the clause in a new environment, constructed by extending the environment part of the closure by a frame in which logical variables have been bound by the unification of the arguments

N.B.

The process of alternating, from left to right, the evaluation and unification of arguments introduces additional expressive power in the model, by allowing variables to get bound before the evaluation of functions (as illustrated in Section 1.3). Still, this process could be replaced by a somewhat simpler evaluation scheme, whereby the two argument lists would be entirely evaluated before unification. According to the evaluation rules for function applications, this simpler scheme would be implied by any model in which predicates were to be represented as functions closures. It is not clear, at this point, whether or not the extra expressive power conferred by the more complex 
scheme justifies the possible overhead incurred. The evaluation of terms before their unification cannot however be dispensed with, as it is an essential characteristic of a model allowing to mix terms. Standard compiling techniques will therefore not be applicable to clauses, whose arguments, in any case, have to be evaluated at run time.

A particularity of the computation model, arising from the possibility of referencing unbound variables which may become bound later on (possibly to other unbound variables, and so on), is that the evaluation of any object containing variables must unroll potential chains of bindings (see Section 3 for more details).

Another particularity arises from multiple clauses predicates, whose application evaluation, as outlined above, is a non deterministic process: each choice of a clause may lead to yet another (or possibly many) multiple clauses predicate application from its body, and son on. This leads to a search process. Whereas a breath-first strategy should be preferred, to ensure completeness, the depth-first strategy is generally adopted, as Prolog does, because the simple chronological backtracking it amounts to is easy to implement.

\subsection{Example}

If we reconsider the example of Section 2.1, the beginning of its evaluation process can be described as follows:

to evaluate an application, i.e. ( $\mathrm{p}$ (cons 'a ? $\mathrm{x}$ )),

first evaluate its operator part, i.e. $\mathrm{p}$;

since it evaluates to a defined logical operator,

consider in turn each of its clauses, i.e. (clause (?x) ( $\mathrm{q}(\mathrm{cdr} ? \mathrm{x}))$ ),

and for all its head arguments, i.e. ?x local to the clause,

alternate the evaluation of pairs of arguments, i.e. local ? $\mathrm{x}$ and (cons 'a ? $\mathrm{x}$ ), with the unification of the resulting values, i.e. [?x] and (a. [?x]);

since this unification succeeds, with local ? $\mathrm{x}$ becoming bound to (a. [?x]), evaluate the body of the clause, i.e. (q (cdr ? $\mathrm{x}$ )),

in its own environment extended by a frame in which local ?x is bound (a. [? $\mathrm{x}]$ ), etc...

\section{The Metacircular Evaluator}

The definition of a metacircular evaluator of expressions will now be given. We will follow the abstract syntax method illustrated in [1], access function being left unspecified. Our presentation will rely on a core subset 
of Scheme, augmented with the following procedures:

(new-closure $\exp _{i} j_{i} \operatorname{env}_{i}$ ) returns a closure associating an expression and an environment

(closure? $j \exp i$ ) returns true if $\exp _{i}$ is a closure

(closure-exp jiclosure $i$ ) returns the expression contained in jclosure $i$

(closure-env jiclosure $i$ ) returns the environment contained in iclosure $i$

After looking at the definition of the top level eval function, we will review the major modifications and/or extensions which, with respect to Abelson and Sussman definitions [1], affects the various evaluation functions called in turn by function eval:

\subsection{The Top Level Eval Function}

Here is the definition of eval:

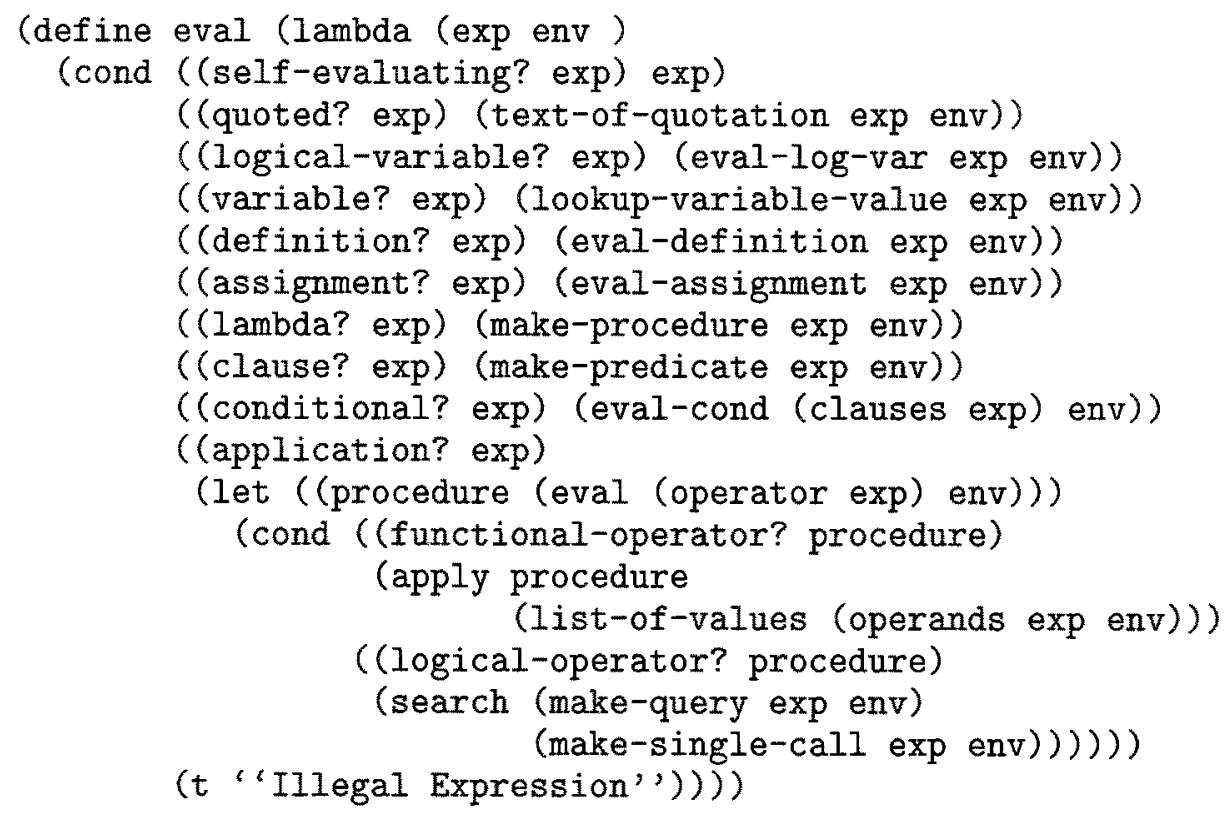

Clearly, this new definition encompasses just two modifications:

- a new case has been introduced to handle occurences of logical variables

- the case for handling applications has been extended to discriminate between standard Scheme procedure applications and logical procedure applications 


\subsection{Evaluation of Quoted Expressions}

Text-of-quotation returns the instantiation (called recursive realization in [7]) of the operand part in the current environment, i.e. each of its logical variables are evaluated in the given environment to unroll potential chains of bindings:

(define text-of-quotation (lambda (exp env)

(instance (car (cdr exp)) env)))

\subsection{Evaluation of Logical Variables}

Eval-log-var, returning the value of a logical variable in a given environment, is clearly a particular case of term instantiation:

(define eval-log-var (lambda (exp env)

(instance exp env)) )

with

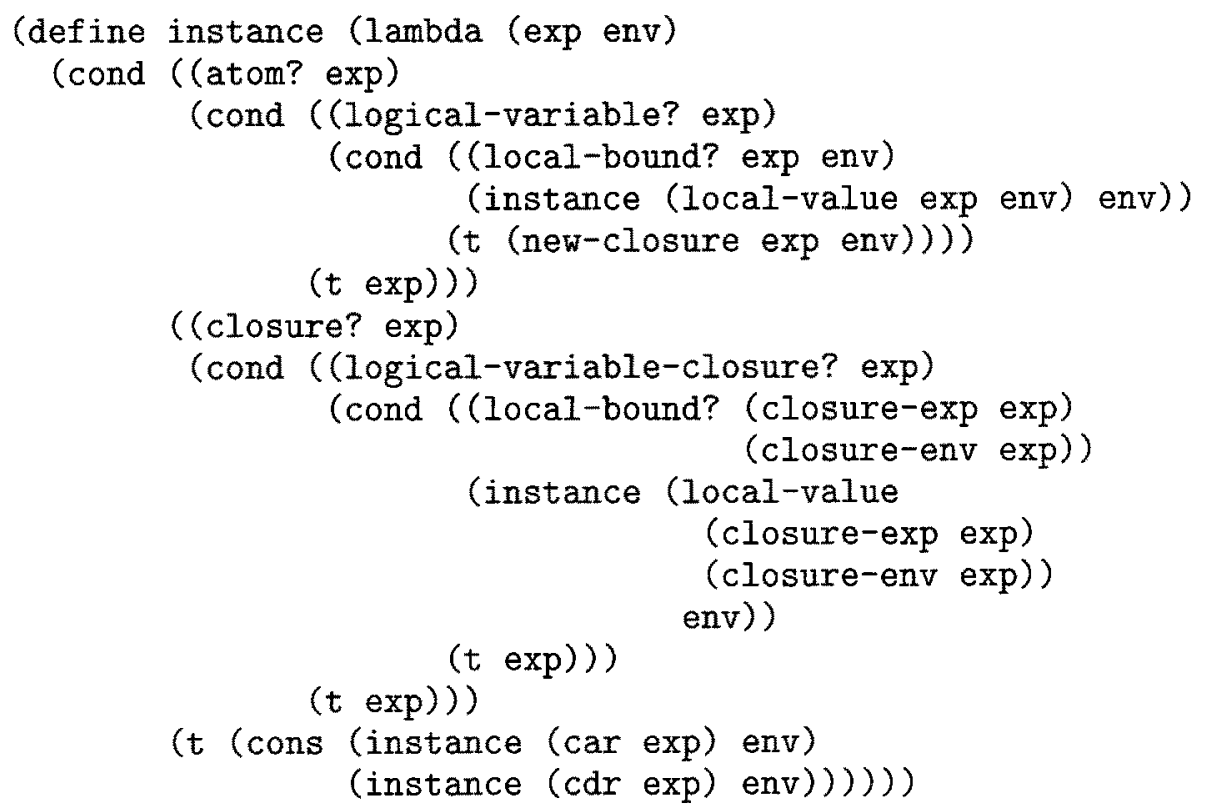

This rather complex definition of the instantiation function reflects the following properties of logical variables: 